\title{
Zentrenbildung beim Maximalversorger - Katalysator der Kommunikation
}

\author{
Creation of Centres in High-Volume Hospitals - \\ Promotor of Communication
}

Autoren

Institut

\author{
C. Möbius, G. Schumacher
}

Klinikum Braunschweig, Chirurgie, Braunschweig, Deutschland

\author{
Schlüsselwörter \\ - Abdominalchirurgie \\ - Zentrum \\ - Zertifizierung \\ - Kommunikation \\ Key words \\ - abdominal surgery \\ - centres of expertise \\ - certification \\ - communication
}

Bibliografie

DOI http://dx.doi.org/

10.1055/s-0031-1283923

Online-Publikation: 21.5.2012

Zentralbl Chir 2013; 138: 53-56

(c) Georg Thieme Verlag KG

Stuttgart · New York

ISSN 0044-409X

Korrespondenzadresse

Privatdozent Dr. med.

Christian Möbius

Klinikum Braunschweig,

Chirurgie

Salzdahlumer Straße 90

38126 Braunschweig

Deutschland

Tel.: $0531 / 5952422$

Fax: $0531 / 5952090$

moebius.christian

@mh-hannover.de

\section{Zusammenfassung \\ $\nabla$}

Die Anzahl der Patienten mit der Diagnose Krebs ist alarmierend: Gemäß der neuen Zahlen des Robert-Koch-Instituts erkranken in Deutschland jährlich ca. 435000 Patienten. 210000 Patienten sterben pro Jahr an dieser Diagnose. Wenn man keinen wesentlichen Fortschritt in der Prävention erreicht, werden im Jahr 2020 rund 25\% mehr Krebsfälle diagnostiziert. In den letzten beiden Jahrzehnten hat es sich durchgesetzt, dass die interdisziplinäre Behandlung von Tumor-Patienten notwendig ist, weil die Vielzahl von unterschiedlichen Therapieoptionen in Abhängigkeit von einer einzelnen Person nicht mehr geleistet werden kann. Das Ziel der Zentrenbildung und der Zertifizierung ist die Etablierung einer möglichst konzentrierten Expertise bei hoher Fallzahl und eine qualitätsgesicherte transparente Therapie. Die zentrale Anforderung an ein Zentrum der Maximalversorgung ist somit die Kommunikation zu ermöglichen, in dem geeignete Rahmenbedingungen geschaffen werden. Neben den räumlichen Möglichkeiten muss ein onkologisches Zentrum die Kommunikation auch fördern, um einen Behandlungskorridor vorzugeben und wesentlich zur Weiterbildung aller Beteiligten beitragen. Zusätzlich hat ein onkologisches Zentrum auch ein Kommunikationsziel, nämlich eine Behandlungsoption für Patienten in jedem Stadium der Erkrankung zu bieten und onkologische Patienten in Studien einzuschließen.

\section{Einleitung \\ $\nabla$}

„[...] Die Zusammenarbeit zwischen den Ärzten in Deutschland ist unzureichend und senkt die Heilungschancen.“ Dieses Zitat stammt aus einem Spiegel Interview in dem Jahr 2000 mit Professor Weißbach, dem damaligen Präsidenten der Deutschen Krebsgesellschaft [1]. Auslöser war die schlechte onkologische Versorgung in Deutsch-

\section{Abstract \\ $\nabla$}

The number of patients with a diagnosis of cancer is alarming: according to new figures from the Robert Koch Institute about 435000 patients are diagnosed annually in Germany. 210000 patients die annually of this diagnosis. Even if no significant progress in prevention has been achieved, in 2020 about $25 \%$ more cases will be diagnosed. In the last two decades it has become established that an interdisciplinary treatment of tumour patients is necessary because the variety of different treatment options depending on an individual person cannot be mastered by one physician. The goal of the centres and certification is to establish a very concentrated expertise in large case numbers and quality-assured transparent treatment. The central requirement of a maximum-care centre is thus to enable communication under appropriate conditions. Promoting not only the space requirements, centre must also develop a treatment corridor and is responsible for further education. Additionally an oncology centre has a goal in communications, namely, to offer a treatment option for all patients in each stage of the disease, and to incorporate cancer patients in clinical trials.

land im internationalen Vergleich und die unzureichende Arbeit der Tumorzentren, welche gemäß einer nationalen Krebskonferenz 1979 etabliert wurden. „Die Idee war, dass ein Tumorpatient dort interdisziplinär diagnostische und therapeutische Angebote bekommt. Doch das ist oft nicht der Fall. Inzwischen haben sich die Tumorzentren überlebt - sie sind zu unbeweglichen Tankern geworden, die sich kaum noch steuern 
lassen." [1] Um der wachsenden Anzahl an Krebserkrankungen zu begegnen und die geforderte interdisziplinäre Qualität zu erreichen, erfolgte im Jahr 2002 eine Evaluation der Tumorzentren unter diesen Gesichtpunkten durch die Unternehmensberatungsfirma Horvath im Auftrag der Deutschen Krebshilfe und der Deutschen Krebsgesellschaft. Zusammenfassend ergab die Evaluation ein sehr heterogenes Ergebnis und deutliche qualitative Unterschiede [2]. Bestätigt werden diese Beobachtungen durch die 2. bundesweite onkologische Qualitätskonferenz. Demnach erhielten im Median nur ca. 55\% der Patienten mit einem Kolonkarzinom im Stadium III eine adjuvante Chemotherapie. Bezogen auf Patienten mit einem Rektumkarzinom im Stadium III erhielten 30-40\% keine Radiochemotherapie [3]. Analysiert man die Daten, bezogen auf das Alter der Patienten, zeigten die Untersuchungen des „cancer trends progress report“, dass die Chemotherapierate bei 20 - 64-Jährigen $80 \%$ beträgt und nur ca. $50 \%$ bei Patienten $>65$ Jahre [3].

Mit dem Ziel, eine flächendeckende, hoch qualitative und interdisziplinäre onkologische Versorgungsstruktur aufzubauen, hat die Deutsche Krebsgesellschaft das Verfahren der zertifizierten Zentrumsbildung ins Leben gerufen [4]. Seither gibt es ein etabliertes Verfahren für alle häufigen Tumorentitäten. Die Anzahl der Organzentren wächst stetig. Im Mittelpunkt dieser Zentren steht die ganzheitliche und interdisziplinäre Therapie eines Krebspatienten. Dieser wird nach Leitlinien-gerechter Diagnostik im Herzstück eines Organzentrums - dem Tumorboard - vorgestellt und ein Behandlungsplan entworfen. Entscheidend ist hier die Kommunikation aller Beteiligten sowohl der unterschiedlichen fachlichen Disziplinen als auch der Vertreter aus unterschiedlichen Sektoren der Krankenversorgung, denn nur ein strukturierter Austausch aller beteiligten Personen kann die medizinische Qualität von Tumorpatienten in allen Phasen ihrer Behandlung nachprüfbar verbessern [5].

Ziel dieser Übersichtsarbeit ist es, die Bedeutung der Kommunikation innerhalb eines Tumorzentrums herauszuarbeiten und die Bereiche der Kommunikation zu definieren.

\section{Methodik \\ $\nabla$}

Zunächst wurden Suchbegriffe ausgewählt, um Referenz-Literatur für diesen Artikel zu identifizieren. Hierzu zählten „Krebs Epidemiologie, Zentrenbildung, Maximalversorger, Zertifizierung, Kommunikation“. Anhand dieser Schlagwörter erfolgte die elektronische Medline-Literaturrecherche (Pub-Med) in dem Zeitraum von 1995 bis 2011. Es konnten somit zahlreiche Artikel in deutscher und englischer Sprache identifiziert werden. Zusätzlich wurden die Erfahrungen ausgewertet, welche die Autoren im Rahmen der Gründung von 4 Organzentren und einem Cancer Center gemacht haben.

\section{Epidemiologie Krebs}

$\nabla$

Die aktuelle Schätzung des Robert-Koch-Instituts für das Jahr 2006 beziffert die Anzahl der Patienten mit der Diagnose Krebs auf 426 800. Insgesamt starben 210000 Menschen an der Diagnose Krebs. Die Frauen sind zum Zeitpunkt der Diagnose Krebs im Mittel 68 und die Männer 69 Jahre alt, jedoch versterben die Frauen an der Diagnose Krebs im Mittel mit 76 und die Männer mit 72 Jahren. Bezogen auf die mittlere Lebenserwartung von derzeit 81 Jahren, bedeutet das ein Verlust von 9Jahren bei Män- nern und 5 Jahren bei Frauen [6]. Vergleicht man die Herz-Kreislauf-Erkrankungen mit den Krebserkrankungen, so beobachtet man einen Rückgang der Sterblichkeit für beide Erkrankungen. Allerdings sinkt die Rate der Neuerkrankungen an Herz-Kreislauf-Erkrankungen und die Rate der Neuerkrankungen an Krebs steigt [7]. In den kommenden Jahrzehnten könnte die Zahl neuer Krebserkrankungen noch deutlicher steigen, weil mit einem wachsenden Anteil älterer Menschen in der Bevölkerung gerechnet werden muss und das Krebsleiden im höheren Lebensalter exponenziell ansteigt. Im Jahr 2020 muss mit ca. 550-600 000 Neuerkrankungen an Krebs gerechnet werden [7]. Mit dem Ziel, sich dieser Entwicklung zu stellen, haben das Bundesministerium für Gesundheit, die Deutschen Krebsgesellschaft, die Deutsche Krebshilfe und die Arbeitsgemeinschaft Deutscher Tumorzentren im Jahr 2008 den Nationalen Krebsplan initiiert [8].

Der Schwerpunkt des Nationalen Krebsplans wurde in der ersten Phase (2009/2010) auf vier Handlungsfelder gelegt: Handlungsfeld 1 ist Weiterentwicklung der Krebsfrüherkennung, Handlungsfeld 2 die Weiterentwicklung der onkologischen Versorgungsstrukturen und der Qualitätssicherung, Handlungsfeld 3 beinhaltet die Sicherstellung einer effizienten onkologischen Behandlung und das Handlungsfeld 4 widmet sich der Stärkung der Patientenorientierung / Patienteninformation ( $\bullet$ Tab. 1).

\begin{tabular}{|c|c|}
\hline $\begin{array}{l}\text { Handlungs- } \\
\text { feld }\end{array}$ & Inhalt \\
\hline 1 & Krebsfrüherkennung \\
\hline 2 & onkologische Versorgungsstruktur und Qualitätssicherung \\
\hline 3 & effiziente onkologische Behandlung \\
\hline 4 & Patientenorientierung und Patientensicherheit \\
\hline
\end{tabular}

\section{Onkologische Versorgungsstrukturen}

Historisch gewachsen gibt es in Deutschland eine strikte Trennung der gesamten Gesundheitsversorgung in Teilgebiete, sogenannte Sektoren [9]. Diese sektorale Aufteilung ist die Grundlage für eine sehr erfolgreiche Arbeit, da sie einen überschaubaren Bereich abbildet und ein hohes Maß an Spezialisierung ermöglicht. Andererseits bedingt diese Sektoralisierung auch eine große Gefahr, wenn der Behandlungserfolg einer Erkrankung die Zusammenarbeit verschiedener Sektoren benötigt. Erhebliche Defizite in der notwendigen Kommunikation und Kooperation führen zu einem schlechten Behandlungsergebnis. Aus diesem Grund steht derzeit die Zentrumsbildung im Mittelpunkt der Etablierung von onkologischen Versorgungsstrukturen [10]. Ziel dieser Zentralisierung ist es, Experten verschiedener Fachrichtungen und Ärzte unterschiedlicher Sektoren zusammenzuführen und gemeinsam die Erkrankung eines Krebspatienten zu diagnostizieren und zu behandeln. Daneben wird zusätzlich eine Zertifizierung angestrebt, um die Diagnostik und Therapie der Patienten an den aktuellen Standards zu messen und die Versorgung flächendeckend zu ermöglichen. Das Ziel der Zertifizierung ist die Etablierung einer qualitätsgesicherten Struktur, welche für die beteiligten Partner und den onkologischen Patienten transparent ist [11]. Durchgesetzt hat sich das 3-Stufen-Modell der Deutschen Krebsgesellschaft ( $\bullet$ Abb.1). Hierbei entspricht der Basis das Organkrebszentrum. In diesem werden häufige Tumorentitäten wie z.B. Darmkrebs oder Brustkrebs gemäß internationaler Thera- 


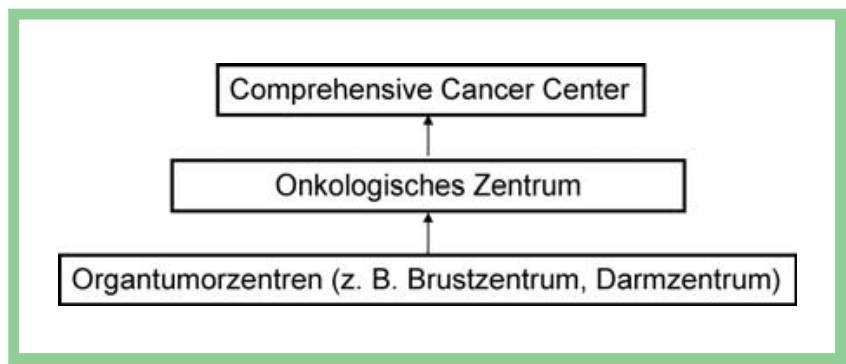

Abb.1 Stufenmodell der Deutschen Krebsgesellschaft zur Etablierung einer onkologischen Versorgungsstruktur in Deutschland.

piestandards ganzheitlich und zu jedem Zeitpunkt der Erkrankung therapiert. Die zweite Stufe der Pyramide bildet das onkologische Zentrum. Hierbei werden verschiedene Organzentren unter einem Dach zusammengefasst. Ziel ist es, die verschiedenen Kräfte einer Region zu bündeln und die onkologische Versorgung einer Region nach hohen Qualitätsansprüchen zu gewährleisten [12].

Die Spitze bildet das „Comprehensive-Cancer-Center“-Programm der Deutschen Krebshilfe. In diesem Programm werden Zentren gefördert, die neben der hochqualitativen Versorgung von Patienten auch translationale Forschung betreiben, also für die rasche Umsetzung neuer Behandlungsmethoden verantwortlich sein sollen [13].

Das Ziel dieser zertifizierten Zentrumsbildung zur Etablierung onkologischer Strukturen ist somit eine hochwertige medizinische Versorgung auf der Basis einer engen Zusammenarbeit unterschiedlicher Fachdisziplinen und unterschiedlicher Sektoren. In diesen Zentren werden moderne Therapiestrategien der Onkologie umgesetzt und sie bilden die Plattform der Kommunikation der Beteiligten untereinander.

\section{Kommunikations-Plattform \\ $\nabla$}

Das onkologische Zentrum bildet eine allumfassende Klammer, in der die einzelnen Organzentren abgebildet sind, Vertreter unterschiedlicher Sektoren des Gesundheitswesens teilhaben und Querschnittsfächer (z.B. Onkologe, Radiologie, Pathologie) vertreten sind. Darüber hinaus koordiniert eine definierte Leitungsstruktur die verschiedenen Aktivitäten. Diese Leitungsstruktur muss eine offizielle Legitimation haben und über ein entsprechendes Budget verfügen [14]. Das kommunikative Herzstück bildet das regelmäßig abgehaltene Tumorboard ( $\boldsymbol{0}$ Abb.2). Dieses ist zwar spezifisch für eine Entität, jedoch bilden insbesondere die Vertreter der Querschnittsfächer und bei speziellen Fragestellungen spezialisierte Fachkräfte (z.B. Metastasenchirurgie) den wesentlichen Grundstock eines Tumorboards. Um eine Kommunikation zu ermöglichen, ist es Aufgabe des Zentrums, hier eine Basis bereitzustellen. Eine Möglichkeit ist ein zentraler Konferenzraum, der die technischen Voraussetzungen zur Präsentation von Untersuchungsergebnissen vorweist und für alle Beteiligten Disziplinen und Vertreter der unterschiedlichen Sektoren gut erreichbar ist. Eine andere Möglichkeit ist das telemedizinische Tumorboard. Hierzu wird vor Beginn einer interdisziplinären Besprechung eine telemedizinische Schaltung zwischen zwei Standorten freigegeben, so dass alle Beteiligten, räumlich getrennt voneinander, onkologische Patienten besprechen können. Neben dem visuellen und auditiven Kontakt aller Beteilig-

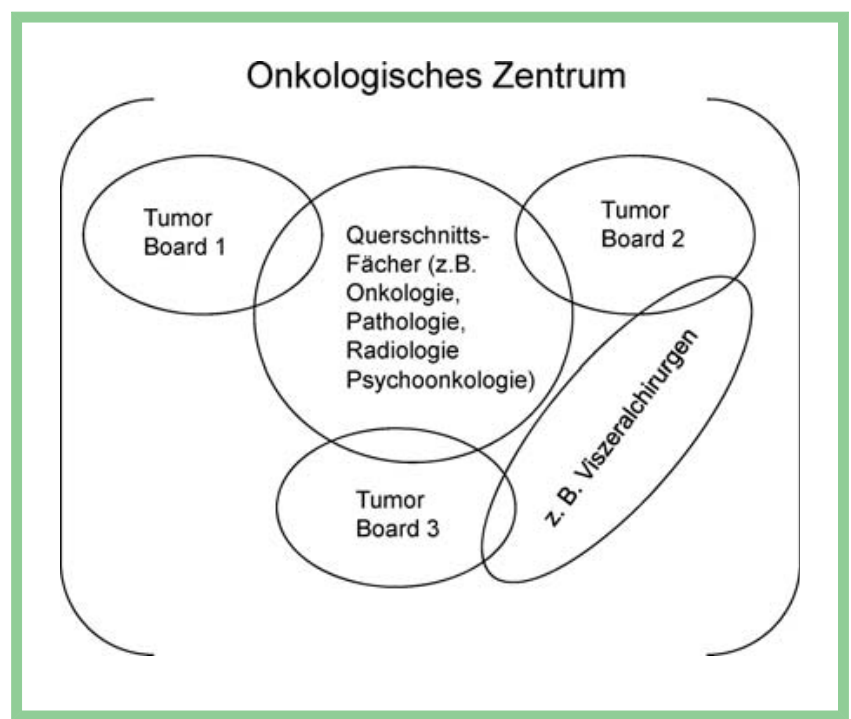

Abb.2 Schnittmengen innerhalb eines onkologischen Zentrums und Plattform der Kommunikation.

ten besteht die Möglichkeit, Bilder (Gastroskopie, CT, MRT, Histologie etc.) und die elektronische Krankenakte zu betrachten. Das telemedizinische Tumorboard ermöglicht somit, verschiedene Standorte eines Krankenhauses miteinander zu verbinden und Kommunikation zu ermöglichen, ohne dass jemand ein Auto bewegen muss. Ein wesentliches Ziel der Zentrumsbildung ist es somit, eine Plattform als zentrale Säule für die interdisziplinäre Kommunikation zu schaffen.

Eine weitere zentrale Säule der erfolgreichen Kommunikation ist die personelle Vorrausetzung. Kommunikation bedeutet nicht nur „sich mitteilen“, sondern auch etwas „Gemeinsames (Behandlungspfad) schaffen“. Vorrausetzung für einen erfolgreichen Behandlungsplan ist die Kenntnis aller Fakten. Hierzu sind die Vertreter der unterschiedlichen Fachrichtungen gefragt. Diese müssen aktiv an der Kommunikation teilnehmen und ihr Spezialwissen zur Verfügung stellen, da nur eine konsensuale Bewertung aller Befunde durch die unterschiedlichen Fachdisziplinen zu einem therapeutischen Konzept führen kann [15]. Auch das ist die Aufgabe eines Zentrums, nämlich entscheidende Personen zu identifizieren und zur Kommunikation zu motivieren.

Die dritte Säule einer erfolgreichen Kommunikation ist die inhaltliche Abstimmung einheitlicher Behandlungspfade, sogenannter „clinical pathways“. Diese werden durch alle beteiligten Personen für die unterschiedlichen Entitäten erarbeitet. Im Fokus steht hier insbesondere die geforderte Diagnostik in Bezug auf die Tumorkategorie („staging“) und die Abklärung eines individuellen Risikoprofils des einzelnen Patienten. Zusätzlich müssen auch psychoonkologische Aspekte (Wille des Patienten, individuelle Erwartungen) berücksichtigt werden [15]. Erst diese Vereinheitlichung ermöglicht eine sinnvolle und zielführende Kommunikation.

\section{Kommunikations-Auftrag \\ $\nabla$}

Neben der Etablierung einer Kommunikations-Plattform und somit der geeigneten Rahmenbedingungen ist es ebenso die Aufgabe eines Zentrums, die Kommunikation aller Beteiligten zu 
beleben. Vorraussetzung für eine qualitätsgesicherte Therapie der onkologischen Patienten ist ein hohes Maß an Spezialisierung und Integration der Versorgung in ein optimales Kommunikations-Netzwerk. Mangelnde Kommunikation, fehlende Effizienz und intersektorale Abschottung führt nachweislich zu regional unterschiedlicher Versorgungsqualität, höherer Morbidität, höherer Mortalität, verminderter Lebensqualität und höheren Behandlungskosten [4]. Das Ziel des onkologischen Zentrums ist somit die Moderation und Lenkung der Kommunikation, um einen interdisziplinär abgestimmten und Leitlinien-gerechten Behandlungskorridor vor Beginn der Therapie für den onkologischen Patienten vorzugeben [16] Abgesehen von dieser zentralen Aufgabe erfüllt das onkologische Zentrum durch die Förderung der Kommunikation auch die Weiterbildung aller beteiligten Personen. Durch das Teilen und Mitteilen von Information und speziellem Wissen (lateinisch communicare) und der Entstehung von etwas Gemeinsamem (lateinisch communio) können die einzelnen Akteure der sehr komplexen und vielschichtigen onkologischen Therapie stetig lernen und sich weiterbilden. Ein weiterer wesentlicher Bestandteil des Kommunikations-Auftrags ist die Öffentlichkeitsarbeit. Ein onkologisches Zentrum soll eine ganze Region prägen und ist verantwortlich für alle onkologischen Patienten und onkologisch tätigen Kollegen in seinem Einzugsgebiet. Kommunikations-fördernd sind in diesem Zusammenhang Kooperationsverträge zwischen Vertretern der unterschiedlichen Gesundheits-Sektoren, um eine genaue Regelung zu treffen, denn alle Beteiligten werden ein Teil ihrer Autonomie zu Gunsten des Zentrums aufgeben müssen [14]. Ein weiterer integraler Bestandteil der Öffentlichkeitsarbeit ist ein „outreach“ Programm. Dieses beinhaltet interdisziplinäre Fortbildungen mit wechselnden Themen und wechselnden Referenten. Neben reinen Vortragsreihen sollten auch interaktive Workshops angeboten werden, um die Kommunikation der Zentrumsteilnehmer und ihrer Zielgruppe zu intensivieren.

\section{Kommunikations-Ziel}

$\nabla$

Im Fokus eines onkologischen Zentrums steht die ganzheitliche Behandlung eines Krebspatienten zu jedem Zeitpunkt seiner Erkrankung. Der Behandlungsplan beginnt mit der qualitätsgesicherten und an Leitlinien orientierten Diagnostik [16]. Wenn alle notwendigen Fakten zu einem Patienten vorliegen, sollen diese von den zuständigen Spezialisten interdisziplinär kommuniziert werden, denn nur in der Zusammenschau aller Befunde ist eine strukturierte Kommunikation und anschließende gemeinsame Entscheidungsfindung möglich. Das Kommunikations-Ziel ist somit ein Behandlungsplan für den Patienten. Im besten Fall kann der onkologische Patient, wenn er die Eingangskriterien erfüllt, in eine Studie eingeschlossen werden. Die hierzu notwendigen Informationen müssen ebenfalls durch das Zentrum kommuniziert und entsprechende organisatorische Strukturen etabliert werden. Wenn eine Studie für den Patienten nicht in Betracht kommt, so muss der Behandlungsplan des Patienten moderne interdisziplinäre Verfahren beinhalten. Ein Beispiel ist hier die multimodale Therapie des Rektumkarzinoms. Daten des
AN-Instituts für Qualitätssicherung in der operativen Medizin an der Otto-von-Guericke-Universität Magdeburg haben zeigen können, dass mit zunehmender Einführung der interdisziplinären Tumorboards auch der Anteil an Patienten mit multimodalen Behandlungsplänen ansteigt [17].

Neben dem Behandlungsplan ist auch die Kommunikation des weiteren Verlaufes ein wesentliches Kommunikationsziel. Innerhalb eines Zentrums müssen z.B. mögliche Komplikationen im Rahmen einer „morbidity-and-mortality“-Konferenz kommuniziert werden. Gerade in einer interdisziplinären Gruppe können Komplikationen ohne persönlichen Bezug objektiv diskutiert werden und führen zu einer stetigen Verbesserung der onkologischen Versorgung. Zusätzlich muss ein Zentrum die NachsorgeDaten der behandelten Patienten erfassen, um eine eigene Qualitätssicherung durchführen zu können und durch die Weitergabe der Daten an ein zentrales Register epidemiologische Aussagen machen zu ermöglichen.

Zusammenfassend kann man sagen, dass ein onkologisches Zentrum an einem Maximalversorger einen vielfältigen Kommunikations-Auftrag hat und umsetzen muss, denn nur der interdisziplinäre und intersektorale Austausch wird der hoch komplexen und qualitätsgesicherten modernen Therapie eines onkologischen Patienten gerecht.

\section{Interessenkonflikt: Nein}

\section{Literatur}

1 Halter $H$, Augstein $R$. Die Ärzte haben versagt. Spiegel Interview mit Professor Weissbach. 2000; 12: 230-234

2 Horvath \& Partners. Evaluierung der Tumorzentren in der BRD 2004. Im Internet: www.tumorzentren.de (Stand 15.11.2011)

3 Schütte J. Unterversorgung in der Onkologie. Onkologe 2008; 14: 701 706

4 Selbmann HK. Bewertung und Zertifizierung von Akut-Krankenhäusern in der Onkologie. Onkologe 2005; 11: 1292-1297

5 Schepp $W$, Heitland $W$. Bedeutung von Zertifizierung und Zentrenbildung in der Viszeralmedizin. Viszeralmedizin 2011; 27: 361-366

6 Robert Koch Institut. Gesundheitsberichtserstattung und Epidemiologie Dachdokumentation Krebs.http://rki.de (Stand 15.11.2011)

7 Junker A, Gaisser A. Immer mehr Krebskranke in Deutschland. Onkologe 2008; 14: 529-534

8 Nationaler Krebsplan. Im Internet: www.bmg.de (Stand 16.11.2011)

9 Lauterbach KW, Stock S, Redaelli M. Struktur und Prozess der Interdisziplinarität in der Onkologie - Disease-Management. Onkologe 2003; 9: $362-367$

10 Blankart CB, Fasten E, Schwintowski HP. Das Deutsche Gesundheitswesen zukunftsfähig gestalten: Patientenseite stärken - Reformunfähigkeit überwinden. Berlin, Heidelberg, New York: Springer; 2009

11 Van Cutsem A, Eijkemanns MJ, Poley JW et al. The multidisciplinary management of gastrointestinal cancer. Best Res Clin Gastroenterol 2007; $21: 1089-1108$

12 Settmacher $U$, Bottke D, Hochhaus A et al. Interdisziplinarität am Beispiel gastrointestinaler Tumore. Onkologe 2009; 15: 1110-1119

13 Wiestler OD. Onkologische Spitzenzentren. Onkologe 2009; 15: 1082 1090

14 Zünkeler M, Schlag PM. Regionale Verbünde in der Onkologie. Onkologe 2009; 15: 1070-1081

15 Siess MA. Tumorzentrum - Tumorboards. Onkologe 2003; 9: 354-361

16 Kopp IB. Von Leitlinien zur Qualitätssicherung. Bundesgesundheitsblatt 2011; 54: 160-165

17 Kube R, Ptok H, Wolff S et al. Quality of medical care in colorectal cancer in Germany. Onkologie 2009; 32: 25-29 